\title{
A TOPOLOGY OF ON/OFF MARX MODULATOR WITH PROTECTION OF LOAD AND SOLID STATE SWITCHES
}

\author{
Anatoly Krasnykh \\ Stanford Linear Accelerator Center \\ 2575 Sand Hill Rd, M/S 33 Menlo Park, California 94025
}

\begin{abstract}
This article discusses a proposal for an ultra fast feedback response that will protect the load and solid state switches of the ON/OFF Marx type modulators. The feedback guards main elements of a modulator against possible arcs in the load, particularly arcs inside of the electron guns. The chief concept behind the proposed response system is an employment of a fraction of the output modulator power as a controlling and guarding pulse during the delivery time. The time constant of the proposed feedback loop lies in the nanosecond range. Peculiarities of proposed topology are discussed.
\end{abstract}

\section{Introduction}

The transformerless topology for modern modulators (see Ref [1] and [2]) is attractive because there is no volt-second limitation for energy delivery from the storage to the load. Additionally, there is no need for a sub-system that aids the efficiency of the ferromagnetic media function. Both features are advantageous and produce an increase in the efficiency and a decrease in the cost, respectively. This approach is materialized by the DTI, Inc. [3] in the context of several SBIR grants sponsored by DoE. Their effort in the realization of the above topology is sufficient and remarkable. The transformerless approach is developed as an alternative to the induction type modulator concept that has been designed at SLAC [4]. The presented here proposal was originally considered for the NLC modulator program. The proposed protection concept may be useful for ILC modulator too.

In this article the progressive idea of load protection is discussed, 'progressive' meaning that the employed feedback loop is fast enough to protect the modulator load and Marx switches. As mentioned earlier, the time constant of proposed feedback loop lies in the nanosecond range. The importance of protecting the RF-devices and solid state type modulators is widely acknowledged. The protection system directly depends on the reliability of the modulator and the lifetime of RF-devices. Disadvantages of the solid state switch usage in high power applications are well known. First, their blocking voltage is less than $10 \mathrm{kV}$. As a result, there is a need to employ hundreds of them to reach the relativistic region where RFsources are efficient enough. The second disadvantage, unfortunately, follows from the first: any uncontrolled load behavior can create unrestorable damage to a whole stack of semiconductors, which are costly, especially for the high power applications. The RF-device can survive and recover for the next pulses; the solid switches, however, are more sensitive to damage than vacuum devices. A reliable protection system is essential for the solid state modulators operation.

\section{Brief Review of Design Considerations}

The protection problem project has been discussed by Dr. S. Gold on $24^{\text {th }}$ IPMS [5]. Additionally, some progress in protection design along with experimental results for the induction type modulator concept [4] was reported on MAC Update 2002 [6]. Installations of the snubber circuit, adjustable spark gap on the secondary HV side of modulator, and modifications on the IGBT drivers to limit $\mathrm{dI} / \mathrm{dt}$ are major elements in the induction concept. The designers pointed out the necessity to work with the IGBT manufactures to improve the $\mathrm{dI} / \mathrm{dt}$ modulator performance. These and other modulator updates allow for fantastic results. The protection topology for the transformerless concept is not discussed. For the TWT solid state Marx type modulator [2], protection circuit has been designed and tested. An idea of 
protection topology was trivial. The TWT current monitor signal was compared with the reference level that represented the normal operation mode. If the signal was suddenly increased, then an error signal was originated, blocking the trigger. The added circuit could control a number of faults without resulting in an otherwise inevitable halt of the Marx modulator run. The delay respond varied according to the amplitude of the error signal. If the pulse amplitude of the beam tube current increased noticeably and suddenly (i.e. there was a heavy discharge in anode-cathode gap) then the minimal time delay was $\sim 0.5-0.7$ usec. In the soft discharge arc the delayed respond is increased to 2.5 usec. Such feedback is appropriate for rather low voltage RF-sources such as the TWT. Conversely, for high power load, such as the high power klystrons, the response time is rather high and a 'progressive' solution is necessary.

\section{Solid State Marx Modulator with the Load and Switch Protection Concept}

Proposed is a topology of the fast feedback load protection for the solid state Marx type modulator where the fraction of the power output is employed as a driving IGBT pulse. The simplest schematic of the solid state Marx type modulator with the load protection is shown in Fig.1.

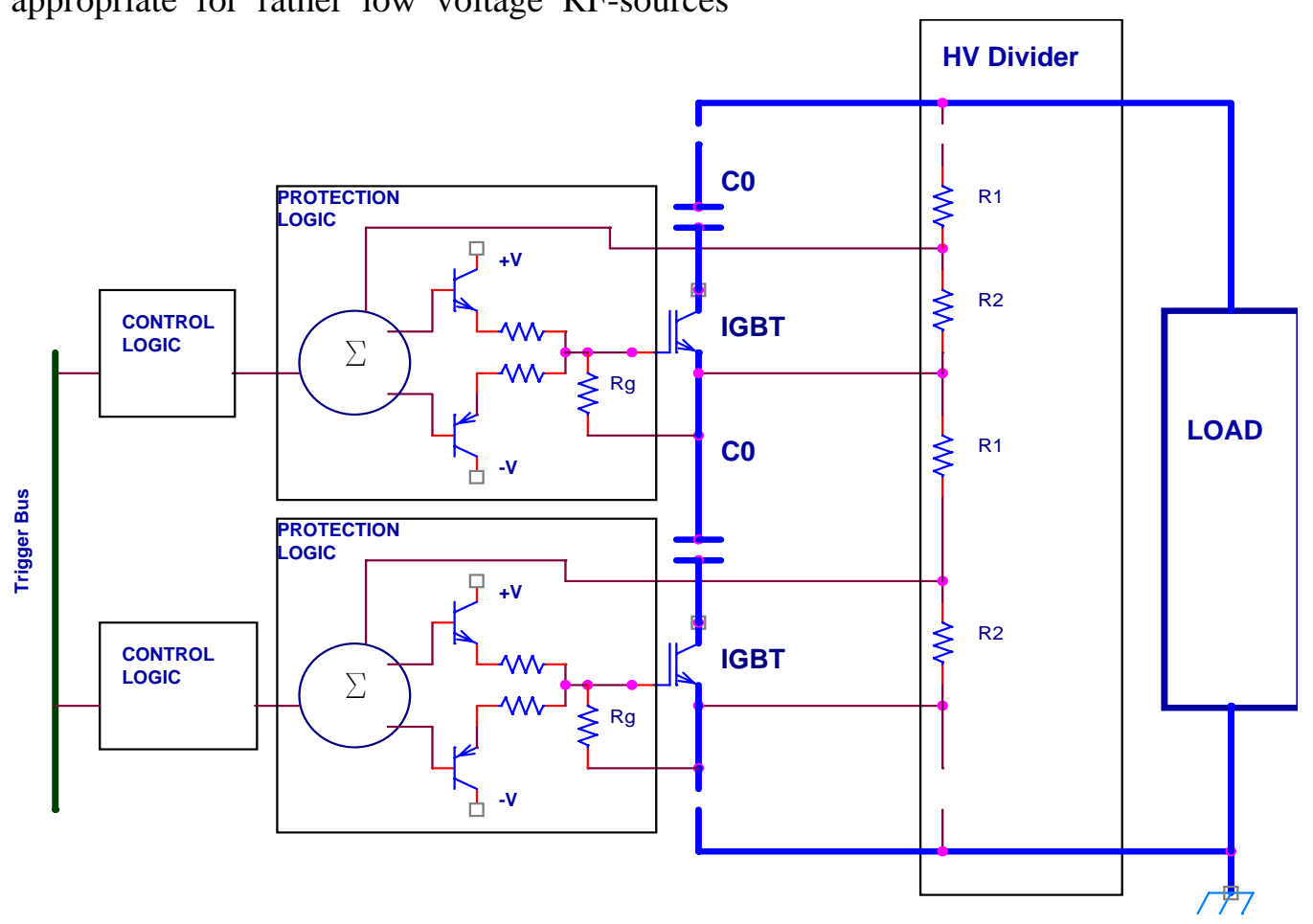

Fig. 1 Circuit Topology of the Solid State Marx with Load Protection

To simplify, the charging elements and power supply are not shown. The alternating series of capacitors C0 and IGBT switches together with the load create a controlled discharge loop. In the feedback circuit contains a high voltage divider, an IGBT with its own driver, and a logic circuit for protection. The high voltage divider contains series $\mathrm{R} 1$ and $\mathrm{R} 2(\mathrm{R} 2<<\mathrm{R} 1)$ resistors, which are placed along the IGBT and C0 stack. The HV divider is connected parallel to the
Marx output. The divider picks up the output voltage and distributes a fraction of the power output along the Marx cells.

The modulator works as follows. In the initial stage all capacitors $\mathrm{C} 0$ are charged up to V0. Each IGBT prevents their discharge at this time. The trigger creates the control signals on the IGBT trigger bus. The amplitude of the driving signal is high enough to turn on each IGBT. The 
pulse duration of the driver pulse is long enough to reach the output voltage pulse plateau. The output voltage rises up to $\mathrm{N}^{*} \mathrm{~V} 0$, where $\mathrm{N}$ is number of the Marx cells. At that moment, the control logic circuit is turned off and the gate voltage starts to be controlled by the feedback circuit. The output voltage controls all IGBT gates during the delivery time. The control logic is activated again to turn the modulator off. There are several ways for a practical realization of control and protection logic circuits. They are not discussed here. If during the delivery time there is an unexpected load impedance drop, the output voltage will immediately react. Due to the fact that each IGBT gate has a short path to the high voltage divider, the modulator switches will respond almost immediately. The limits of the response time depend on the gate RC-time constant. The output driver and protection feedback circuits may be designed in such way that the time constant of the response may be set in the nanosecond range. The proposed concept will inherently limit the deposit arc energy due to high speed sensing. The advantage is due to fast opening before possible damage in the electron device can occur. Note that the driver IGBT function in the proposed topology is different in comparison with the usual Marx driver. The driver circuit works for the rise and the slope of the pulse only. The plateau of the output power is secured by the feedback circuit. The switch and load protections for the front and the slope of the pulse can be designed in the usual way: to diagnose overcurrent against reference level and to open the individual IGBT if there is an imbalance. This method has been studied by author for the TWT Marx modulator [7].

The possible difficulties of the proposed topology can arise if the reliability of the high voltage divider is low. It must be designed with a low field gradient and high stable parameters. The broadband of this divider has to be higher than the inverse rise time of the output voltage. The efficiency of the modulator with the load protection circuit will be slightly less than without one because a part of the energy is used to keep the IGBT switches in the 'ON' mode. The reduction in efficiency depends on the ratio of the load impedance and the high voltage divider. The evaluation of the efficiency reduction for the hypothetic NLC Marx type modulator is less than $3 \%$. The incretion into the Marx Bank of the high voltage divider will increase the parasitic capacity. The Marx architecture, proposed in Ref [3], can mitigate this effect. The proposed topology been modeled by the OrCAD code. Results of the simulations prove the workability of the discussed topology.

\section{Conclusion}

The proposed protection system of the Marx Bank is discussed. A major advantage of topology is a fact the load and solid state switches are safeguarded during the delivery time by a fraction of the output pulse. The nanosecond rage of the time constant in feedback loop allows for immediate response, preventing damage to both the load and modulator. The concept would require a more detailed design and practical realization.

This work is supported by the U.S. DoE under contract \#DE-AC03-76SF00515

\section{Reference:}

[1] A. Krasnykh, et al. A Solid State Marx Modulator for Driving a TWT, Conference Record of the $24^{\text {th }}$ IPM Symposium, June 26-29, 2000, Norfolk, Virginia, p. 209

[2] A. Krasnykh, Analyses of Klystron Modulator Approaches for NLC, Proc. of LINAC 2000, Monterey, California, p. 772 [3] www.divtecs.com/papers/ [Online available] [4] R. Cassel, et al. Solid State Induction Modulator replacement for SLAC Klystron Modulators, Conference Record of the $24^{\text {th }}$ IPM Symposium, June 26-29, 2000, Norfolk, Virginia, p. 157

[5] S. Gold, Thyratron-PFN, IGBT Hybrid, and Direct Switched Modulator R\&D As it Effects

Klystron Protection, Conference Record of the $24^{\text {th }}$ IPM Symposium, June 26-29, 2000, Norfolk, Virginia, p. 5

[6] www.project.slac.stanford.edu/lc/local/ MAC/NOV2002/MAC_Cassel_1106.pdf

[Online available]

[7] S. Gold, SLAC-PUB-10435, April 2001 